\title{
Assessment of thermotactile and vibrotactile thresholds for detecting sensorineural components of the hand-arm vibration syndrome (HAVS)
}

\author{
Ying $\mathrm{Ye}^{1} \cdot$ Michael J. Griffin ${ }^{1}$
}

Received: 12 April 2017 / Accepted: 11 September 2017 / Published online: 16 September 2017

(C) The Author(s) 2017. This article is an open access publication

\begin{abstract}
Background Thermotactile thresholds and vibrotactile thresholds are measured to assist the diagnosis of the sensorineural component of the hand-arm vibration syndrome (HAVS).

Objectives This study investigates whether thermotactile and vibrotactile thresholds distinguish between fingers with and without numbness and tingling.

Methods In 60 males reporting symptoms of the hand-arm vibration syndrome, thermotactile thresholds for detecting hot and cold temperatures and vibrotactile thresholds at 31.5 and $125 \mathrm{~Hz}$ were measured on the index and little fingers of both hands.

Results In fingers reported to suffer numbness or tingling, hot thresholds increased, cold thresholds decreased, and vibrotactile thresholds at both 31.5 and $125 \mathrm{~Hz}$ increased. With sensorineural symptoms on all three phalanges (i.e. numbness or tingling scores of 6), both thermotactile thresholds and both vibrotactile thresholds had sensitivities greater than $80 \%$ and specificities around $90 \%$, with areas under the receiver operating characteristic curves around 0.9. There were correlations between all four thresholds, but cold thresholds had greater sensitivity and greater specificity on fingers with numbness or tingling on only the distal phalanx (i.e. numbness or tingling scores of 1) suggesting cold thresholds provide better indications of early sensorineural disorder.
\end{abstract}

Michael J. Griffin

M.J.Griffin@soton.ac.uk

1 Human Factors Research Unit, Institute of Sound and Vibration Research, University of Southampton, Southampton, England SO17 1BJ, UK
Conclusions Thermotactile thresholds and vibrotactile thresholds can provide useful indications of sensorineural function in patients reporting symptoms of the sensorineural component of HAVS.

Keywords Vibrotactile perception thresholds . Thermotactile thresholds · Hand-transmitted vibration . Hand-arm vibration syndrome $\cdot$ Sensorineural dysfunction

\section{Introduction}

Prolonged occupational exposure to hand-transmitted vibration has been associated with disorders in the vascular, neurological, and musculoskeletal structures of the human hand-arm system, collectively called the 'hand-arm vibration syndrome' (HAVS) (Griffin 1990). Vibration-induced neuropathy in the hand often manifested as reduced sensitivity, numbness, pain, tingling, or clumsiness in hand movements can reduce work ability and the quality of life (Anonymous 1995). It has been suggested that peripheral sensorineural symptoms may cause more discomfort and disability than vibration-induced vascular disorders, such as white finger, since this latter is episodic (usually triggered by exposure to cold) while sensory disturbances can be persistent and may interfere with life activities including sleep (Lundborg et al. 1990).

The hand-arm vibration syndrome is currently staged for severity according to the Stockholm Workshop scales for vascular disorders (Gemne et al. 1987) and sensorineural disorders (Brammer et al. 1987). These staging systems compound a mixture of signs and symptoms. In respect of sensorineural disorders, it is not clear what type of numbness or tingling is required or how reduced sensory perception, reduced tactile discrimination, or reduced manipulative 
dexterity should be measured, or what degree of reduction is required for a positive diagnosis (Griffin 2008). Consequently, there is no gold standard test to diagnose any stage of the sensorineural component of HAVS and many alternative tests have been suggested. In the UK, thermal thresholds and vibrotactile thresholds are recommended for assessing changes in sensorineural function associated with exposures to hand-transmitted vibration (Lindsell and Griffin 1998).

The perception of temperature is mediated by hot and cold receptors via unmyelinated C-fibres and thin myelinated A- $\delta$ fibres, respectively (Dyck et al. 1993). It has been reported that these nerve fibres can be damaged by occupational exposure to hand-transmitted vibration (e.g. Hirosawa et al. 1992). Currently, there is no internationally standardised method for the measurement and evaluation of thermotactile thresholds, although recommendations for thermal testing procedures have been provided by national academies, scientific associations, and research networks (Lindsell and Griffin 1998; Shy et al. 2003; Chong and Cros 2004; Rolke et al. 2006).

The perception of vibration is mediated by various mechanoreceptors via large-diameter myelinated A- $\alpha$ afferent nerve fibres (Burgess and Perl 1973). Using suitable apparatus and appropriate frequencies of vibration, the perception of vibration mediated by two different mechanoreceptors (Meissner's and Pacinian corpuscles) can be determined. Various studies have concluded that the sensitivity of mechanoreceptors is reduced by occupational exposure to handtransmitted vibration (Lundström et al. 1999; Bovenzi et al. 2011; Poole et al. 2016). The measurement of vibrotactile thresholds has been standardised in ISO 13091-1 (2001), and normative thresholds from a few studies have been suggested (ISO 13091-2 2003).

Both thermotactile thresholds and vibrotactile thresholds have been used to assist the diagnosis of disorders in patients reporting sensorineural symptoms of occupational origin (e.g. exposure to hand-transmitted vibration, industrial solvents, acrylamide) and non-occupational origin (e.g. diabetes, uraemia, and toxic-, infectious- or immune-associated neuropathies) (Zaslansky and Yarnitsky 1998; Shy et al. 2003; Chong and Cros 2004). The thresholds can be determined non-invasively using psychophysical methods to assess the integrity of the neuroanatomic pathway between the peripheral receptors and the sensory cortex. From a comparison of four thresholds (i.e. hot, cold, Meissner's, and Pacinian), it is possible to identify whether any deterioration in perception is concentrated in one or more sensory unit or in one or more neural pathway.

For a group of males reporting symptoms of the hand-arm vibration syndrome, this paper compares thermotactile and vibrotactile thresholds between fingers with symptoms of numbness or tingling and fingers without symptoms of numbness or tingling. The tests were performed in accord with the HSE recommended procedure (Lindsell and Griffin 1998). It was hypothesised that there would be increases in vibrotactile thresholds at 31.5 and $125 \mathrm{~Hz}$ and increases in hot thresholds but decreases in cold thresholds in fingers reported to suffer numbness and tingling. It was further hypothesised that there would be positive correlations between the hot thresholds and vibrotactile thresholds and negative correlations between cold thresholds and vibrotactile thresholds, indicating that fingers more affected according to one test tend to be more affected according to another test.

\section{Methods}

\section{Apparatus}

Thermotactile thresholds (HVLab thermal aesthesiometer, University of Southampton).

An HVLab thermal aesthesiometer was used to measure thermotactile thresholds (hot and cold thresholds) via the method of limits (see Table 1). Thresholds were measured on the distal phalanx of the index and little fingers of the right and left hands.

Subjects placed their fingertips so that the centre of the distal phalanx coincided with the centre of the applicator surface. They were instructed to apply a constant finger force of $2 \mathrm{~N}$ to the applicator surface, which they monitored using a digital scale located below the applicator. The temperature of the applicator increased or decreased from a reference temperature of $32.5^{\circ} \mathrm{C}$ at a rate of $1{ }^{\circ} \mathrm{C} / \mathrm{s}$. Subjects were instructed to press the response button as soon

Table 1 Parameters of the HVLab thermal aesthesiometer and $H V L a b$ vibrotactile perception meter

\begin{tabular}{ll}
\hline Parameter & Condition \\
\hline $\begin{array}{l}\text { Thermotactile thresholds } \\
\text { Contact area }\end{array}$ & Circular, $5.5 \mathrm{~cm}$ diameter \\
Contact surface & Smooth and planar \\
Psychophysical method & Method of limits \\
Number of judgements & Six hot or cold \\
Reference temperature & $32.5^{\circ} \mathrm{C}$ \\
Rate of change in temperature & $1{ }^{\circ} \mathrm{C} / \mathrm{s}$ \\
Vibrotactile thresholds & \\
Probe diameter & $6 \mathrm{~mm}$ \\
Probe surround gap & $2 \mathrm{~mm}$ \\
Contactor surface & $\mathrm{Smooth}$ and planar \\
Psychophysical method & von Békésy \\
Number of judgements & $\mathrm{At} \mathrm{least} \mathrm{six} \mathrm{peaks} \mathrm{and} \mathrm{troughs}$ \\
Rate of change in stimulus & $3 \mathrm{~dB} / \mathrm{s}$ \\
Push force & $2 \mathrm{~N}$
\end{tabular}


as they perceived a change in temperature (i.e. increase or decrease in temperature). The temperature of the applicator then returned to the reference temperature and was held at $32.5{ }^{\circ} \mathrm{C}$ for a random interval before the temperature increased or decreased again.

Six hot and six cold thresholds were determined. For both thresholds, the mean was calculated from the last four judgements. The temperature difference between the hot threshold and the cold threshold, known as the thermal neutral zone, was also calculated on all four fingers.

Vibrotactile thresholds (HVLab vibrotactile perception meter, University of Southampton).

An $H V L a b$ vibrotactile perception meter was used to measure vibrotactile thresholds (thresholds at 31.5 and $125 \mathrm{~Hz}$ ) via the von Békésy method in a manner compliant with the methods in ISO 13901-1 (2001) (see Table 1). Thresholds were measured on the distal phalanx of the index and little fingers of the right and left hands.

Subjects were instructed to place their fingertip so that the centre of the distal phalanx coincided with the centre of the probe of the applicator. The magnitude of vibration on the applicator increased from zero at the start of the test. Subjects were instructed to press and hold the response button down as soon as they perceived a vibration sensation and to release the response button as soon as they did not perceive the vibration.

Measurements were taken for a minimum of six reversals over at least $45 \mathrm{~s}$, and the mean was calculated from all the peaks and troughs with the exception of the first peak and first trough.

\section{Subjects}

Sixty male patients referred to the Human Factors Research Unit (University of Southampton) for Tier 5 testing for the hand-arm vibration syndrome agreed to participate in the study (Lindsell and Griffin 1998). This study reports findings from all 60 successive participants (i.e. there were no exclusions). The patients were medicolegal referrals and employer referrals.

The tiered system of health surveillance currently used in the UK has five stages (short questionnaire prior to work, annual questionnaire surveillance, HAVS assessment by an occupational nurse, diagnosis by occupational physician, and objective testing for signs of HAVS) (Health and Safety Executive 2005).

All 60 patients participating in the study had a history of smoking, but eight patients were not currently smoking. Fifty-three patients reported regular drinking of alcohol, five reported long-term medications, 12 had been exposed to chemicals at the workplace, and four had injury or surgery of their hands. Only one patient had noticed a change in skin quality (rough and dry).
They had their thermotactile thresholds and vibrotactile thresholds measured as part of their assessment. The subjects were in the clinic at a constant ambient temperature of $21 \pm 1{ }^{\circ} \mathrm{C}$, with $40-45 \%$ humidity and no noticeable air flow, for more than $1 \mathrm{~h}$ before the tests commenced.

All patients were right handed and with a history of occupational use of hand-held vibratory tools: a mean exposure of 23 years (SD: 6.8, range 5-45 years). They had used a wide range of vibratory tools in various jobs (e.g. gardener, maintenance worker, welder, fitter) with daily durations of exposure that varied between jobs. The subjects completed a health questionnaire and gave their written informed consent to participate in the study. The mean age of the patients was 52.9 years (SD: 12.4 , range $30-70$ years), their mean stature was $176.8 \mathrm{~cm}$ (SD: 5.7, range 165-190 cm), their mean weight was $83.1 \mathrm{~kg}$ (SD: 11.8 , range $63-126 \mathrm{~kg}$ ), and their mean body mass index (BMI) was 26.6 (SD: 5.7, range 19.6-38.8).

The subjects were requested to avoid consuming caffeine for $4 \mathrm{~h}$ and alcohol for $12 \mathrm{~h}$ prior to the testing. The study was approved by the Ethics Committee of the Faculty of Engineering and the Environment (Application number 10704).

\section{Procedure}

Initially, patients were questioned about their occupational history, social and medical history, and symptoms (including any finger whiteness, numbness, tingling, and muscular problems). The locations of any finger blanching, numbness, or tingling were mapped using the scoring system (Griffin 1990, 2008). All decisions to include or exclude a symptom were made when identifying symptoms during interview prior to testing thresholds and therefore prior to data analysis.

The following tests were then performed in sequence for Tier 5 HAVS assessment: Purdue pegboard, grip strength, thermotactile thresholds, vibrotactile thresholds, finger rewarming, and finger systolic blood pressures. All tests were obtained using a computer with professional diagnostic software. The results were calculated, and diagnostic criteria were applied automatically within the software to minimise any bias introduced by the experimenter. The associations between vascular symptoms and signs in these patients have been reported elsewhere (Ye and Griffin 2016a).

\section{Statistical methods}

Data analysis was performed using the software package SPSS (version 22.0). The data were summarised with the median as a measure of central tendency and the inter-quartile range as a measure of dispersion. Nonparametric tests were employed to analyse the data, which were not normally 
distributed. The Wilcoxon matched-pairs signed ranks test was used to investigate differences in finger skin temperatures before the measurement of thermotactile thresholds and vibrotactile thresholds. The Mann-Whitney $U$ test was used to investigate differences between groups: fingers with and without symptoms of finger numbness or tingling. The Spearman rank correlation coefficient was used to investigate associations between thermotactile thresholds (hot and cold) and vibrotactile thresholds (at 31.5 and $125 \mathrm{~Hz}$ ). The diagnostic criteria used in the study were: (1) hot thresholds greater than $45{ }^{\circ} \mathrm{C}$ and cold thresholds lower than $22{ }^{\circ} \mathrm{C}$ and (2) vibrotactile thresholds greater than $0.3 \mathrm{~m} / \mathrm{s}^{2}$ r.m.s. at $31.5 \mathrm{~Hz}$ and greater than $0.7 \mathrm{~m} / \mathrm{s}^{2}$ r.m.s. at $125 \mathrm{~Hz}$. Receiver operating characteristic (ROC) analysis showed the effects of varying these criteria. The diagnostic accuracies of thermotactile thresholds and vibrotactile thresholds were investigated by calculating the areas under the ROC curves (AUC). The diagnostic criteria used in the study are shown in Table 2. The 'possible dysfunction' and 'definite dysfunction' (i.e. cut-off) values are one standard deviation and 2 standard deviations greater than mean values for healthy subjects reported previously (see Lindsell and Griffin 1998, 2002). The normative data of Lindsell and Griffin (2002) were obtained from 237 subjects in ten studies with 80 white collar workers and 157 blue collar workers within the UK. All subjects were male and of working age. No subjects reported symptoms of blanching, numbness, or tingling. No other medical conditions were reported.

The criterion for statistical significance was $p<0.05$. The reported $p$ values have been adjusted for multiple comparisons.

\section{Results}

The four tested fingers (index and little fingers on right and left hands) of the 60 patients reporting symptoms

Table 2 Diagnostic criteria for hot and cold thresholds and vibrotactile thresholds at 31.5 and $125 \mathrm{~Hz}$

\begin{tabular}{lll}
\hline Test & $\begin{array}{l}\text { Possible dys- } \\
\text { function }\end{array}$ & $\begin{array}{l}\text { Definite } \\
\text { dysfunc- } \\
\text { tion }\end{array}$ \\
\hline Hot threshold $\left({ }^{\circ} \mathrm{C}\right)$ & $>45$ & $>48.5$ \\
Cold threshold $\left({ }^{\circ} \mathrm{C}\right)$ & $<22$ & $<19$ \\
$31.5 \mathrm{~Hz}$ threshold $\left(\mathrm{m} / \mathrm{s}^{2}\right.$, r.m.s. $)$ & $>0.3$ & $>0.4$ \\
$125 \mathrm{~Hz}$ threshold $\left(\mathrm{m} / \mathrm{s}^{2}\right.$, r.m.s. $)$ & $>0.7$ & $>1.0$ \\
\hline
\end{tabular}

Possible dysfunction is identified when the measured threshold differs from thresholds measured in a reference group of males without symptoms by more than the mean plus one standard deviation, and definite dysfunction is assumed when the difference is greater than the mean plus two standard deviations (Lindsell and Griffin 1998, 2002) of the hand-arm vibration syndrome were categorised into two groups depending on the symptoms reported: Group A-fingers with sensorineural symptoms (i.e. fingers reported to have numbness or tingling) and Group B-fingers without sensorineural symptoms (i.e. fingers reported to be without numbness and without tingling). Temporary numbness and tingling can occur without a neurological disorder (e.g. as a normal response to vibration and other factors), so temporary numbness or tingling after exposure to hand-transmitted vibration, after gripping, or only associated with cold was excluded. Reports of numbness and tingling were not symmetrical and the areas affected differed between numbness and tingling. A 'sensorineural score' was calculated as the greatest of the score reported for numbness and the score reported for tingling, with a score of 1 for symptoms on the distal phalanx, 2 for the middle phalanx, and 3 for the proximal phalanx (Griffin 1990). For example, if a finger had a 'numbness score' of 6 (numbness on all three phalanges) and a 'tingling score' of 1 (tingling on the distal phalanx), the 'sensorineural score' was 6 . The symptoms differed between fingers, so the number of fingers in Group A and Group B varied between fingers. Of the 240 fingers tested, 154 fingers were reported to have sensorineural symptoms (144 fingers had numbness, 131 fingers had tingling, and 154 had either numbness or tingling or both numbness and tingling) and 136 fingers were reported to have vascular symptoms (i.e. finger blanching provoked by cold conditions).

Statistical analysis has been performed on sensorineural scores reported between fingers within subjects; there was no significant correlation between symptoms reported on different fingers within subjects ( $p=0.108-0.377$, Spearman).

The finger skin temperatures measured prior to measuring thermotactile thresholds did not differ from the temperatures measured prior to measuring vibrotactile thresholds on either the right or left hands ( $p=0.156-0.387)$.

There were lower baseline finger skin temperatures on fingers with sensorineural symptoms (Group A, median: $27.2^{\circ} \mathrm{C}$, range $23.8-32.4^{\circ} \mathrm{C}$ ) compared with fingers without symptoms (Group B, median: $28.8^{\circ} \mathrm{C}$, range $25.1-34.1^{\circ} \mathrm{C}$ ) $(p=0.006-0.027)$.

\section{Thermotactile thresholds}

The medians and inter-quartile ranges of hot thresholds and cold thresholds on fingers in Group A and Group B are shown in Table 3. The neutral zones are also shown in Table 3.

There were no significant differences in either hot or cold thresholds between the four fingers (i.e. four locations) in either Group A or Group B ( $p=0.132-0.476)$. The hot thresholds were higher in Group A than in Group 
Table 3 Medians (and inter-quartile ranges) of hot thresholds, cold thresholds, neutral zones, and vibrotactile thresholds at 31.5 and $125 \mathrm{~Hz}$ on the distal phalanges of fingers with sensorineural symptoms (Group A) and fingers without sensorineural symptoms (Group B)

154 fingers with any sensorineural symptom (Group A)

\section{Hot thresholds $\left({ }^{\circ} \mathrm{C}\right)$}

Cold thresholds $\left({ }^{\circ} \mathrm{C}\right)$

Neutral zone $\left({ }^{\circ} \mathrm{C}\right)$

Thresholds, $31.5 \mathrm{~Hz}$ (m/ $/ \mathrm{s}^{2}$ r.m.s.)

Thresholds, $125 \mathrm{~Hz}$ (m/s $\mathrm{s}^{2}$ r.m.s.)

$48.2(45.7-50.9)^{* *}$
$16.8(14.2-21.3)^{* *}$
$30.6(24.4-32.7)^{* *}$
$0.41(0.34-0.59)^{* *}$
$1.19(0.86-1.72)^{* *}$

$* p<0.01, * * p<0.001$ : significant increase in hot threshold or decrease in cold thresholds on fingers with sensorineural symptoms compared to fingers without sensorineural symptoms (Mann-Whitney $U$ test)
B $(p<0.01)$. The cold thresholds were lower in Group A than in Group B $(p<0.001)$. The thermal neutral zone was greater in fingers with sensorineural symptoms than in fingers without sensorineural symptoms $(p<0.001)$. The mean difference (with $95 \%$ confidence interval) between fingers with and without sensorineural symptoms for hot thresholds was 6.91 (CI of 5.81-8.01) with an effect size of 1.66 (CI of 1.35-1.96). The mean differences (with $95 \%$ confident intervals) between fingers with and without sensorineural symptoms for cold thresholds and for the neutral zone were 8.63 (CI of 7.87-9.39) and 15.52 (CI of 13.98-17.06), with effect sizes of 2.59 (CI of 2.23-2.93) and 2.68 (CI of 2.31-3.02), respectively.

On fingers with sensorineural symptoms (i.e. Group A), a greater change in threshold for the detection of hot and cold temperatures was found on fingers with a sensorineural score of 6 than on fingers with a sensorineural score of 1 or $3(p<0.01)$. There were no significant differences in either hot thresholds or cold thresholds between fingers with sensorineural scores of 1 and 3 ( $p=0.217-0.829)$.

The finger skin temperatures measured prior to the measurement of thermotactile thresholds were not correlated with the hot or cold thresholds at any of the four locations in either Group A or Group B ( $p=0.128-0.635)$.

The sensitivities and specificities of the thermotactile thresholds to distinguish between fingers with and without sensorineural symptoms were 81 and $92 \%$ for hot thresholds, 87 and $94 \%$ for cold thresholds, and 77 and $88 \%$ for the neutral zone. The area under the receiver operating characteristic curve and $95 \%$ confidence intervals (CI) were 0.89 (CI of 0.81-0.97) for hot thresholds, 0.96 (CI of 0.92-1.00) for cold thresholds, and 0.81 (CI of $0.75-0.87$ ) for the neutral zone. The diagnostic odds ratio (DOR) and $95 \%$ confidence intervals for hot thresholds, cold thresholds, and the neutral zone were 47.0 (CI of 20-112), 107 (CI of 38-296) and 25 (CI of 12-53).

The ROC curves for sensorineural symptoms calculated for hot and cold thresholds and vibrotactile thresholds at 31.5 and $125 \mathrm{~Hz}$ are shown in the upper part of Fig. 1. The ROC curves are shown separately according to the severity of the reported symptoms. The corresponding ROC curves for any symptoms of finger blanching (scores of 1,3 , or 6 ) calculated for hot and cold thresholds and vibrotactile thresholds at 31.5 and $125 \mathrm{~Hz}$ are shown in the lower part of Fig. 1.

\section{Vibrotactile thresholds}

The medians and inter-quartile ranges of vibrotactile thresholds measured on the index and little fingers of the right and left hands at 31.5 and $125 \mathrm{~Hz}$ in Group A and Group B are shown in Table 3.

There were no significant differences in vibrotactile thresholds at either 31.5 or $125 \mathrm{~Hz}$ between the four fingers (i.e. four locations) in either Group A or Group B $(p=0.212-0.539)$. The vibrotactile thresholds at 31.5 and $125 \mathrm{~Hz}$ were significantly higher in Group A than in Group B $(p<0.001)$. The mean differences (with $95 \%$ confident intervals) between fingers with and without sensorineural symptoms for vibrotactile thresholds at 31.5 and $125 \mathrm{~Hz}$ were 0.31 (CI of 0.26-0.36) and 0.80 (CI of 0.62-0.98), with effect sizes of 1.73 (CI of 1.42-2.03) and 1.16 (CI of $0.87-1.44)$, respectively.

On fingers with sensorineural symptoms (i.e. in Group A), vibrotactile thresholds at both 31.5 and $125 \mathrm{~Hz}$ were greater on fingers with sensorineural scores of 3 and 6 than on fingers with a sensorineural score of $1(p<0.05)$. There were no significant differences in thresholds at 31.5 and $125 \mathrm{~Hz}$ between fingers with sensorineural scores of 3 and $6(p=0.092-0.418)$.

The finger skin temperatures prior to measuring the vibrotactile thresholds were not correlated with vibrotactile thresholds at $31.5 \mathrm{~Hz}$ at any of the four locations in either Group A or Group B ( $p=0.148-0.335)$. However, there was a negative correlation between finger skin temperatures and vibrotactile thresholds at $125 \mathrm{~Hz}$ at all four locations in Group B ( $p<0.05)$, but not in Group A ( $p=0.418-0.571)$.

The sensitivities and specificities of the vibrotactile thresholds to distinguish between fingers with and without sensorineural symptoms were 80 and $91 \%$ for thresholds at $31.5 \mathrm{~Hz}$ and 82 and $91 \%$ for thresholds at $125 \mathrm{~Hz}$. The AUC and $95 \%$ confidence intervals were 0.90 (CI of $0.84-0.96$ ) 


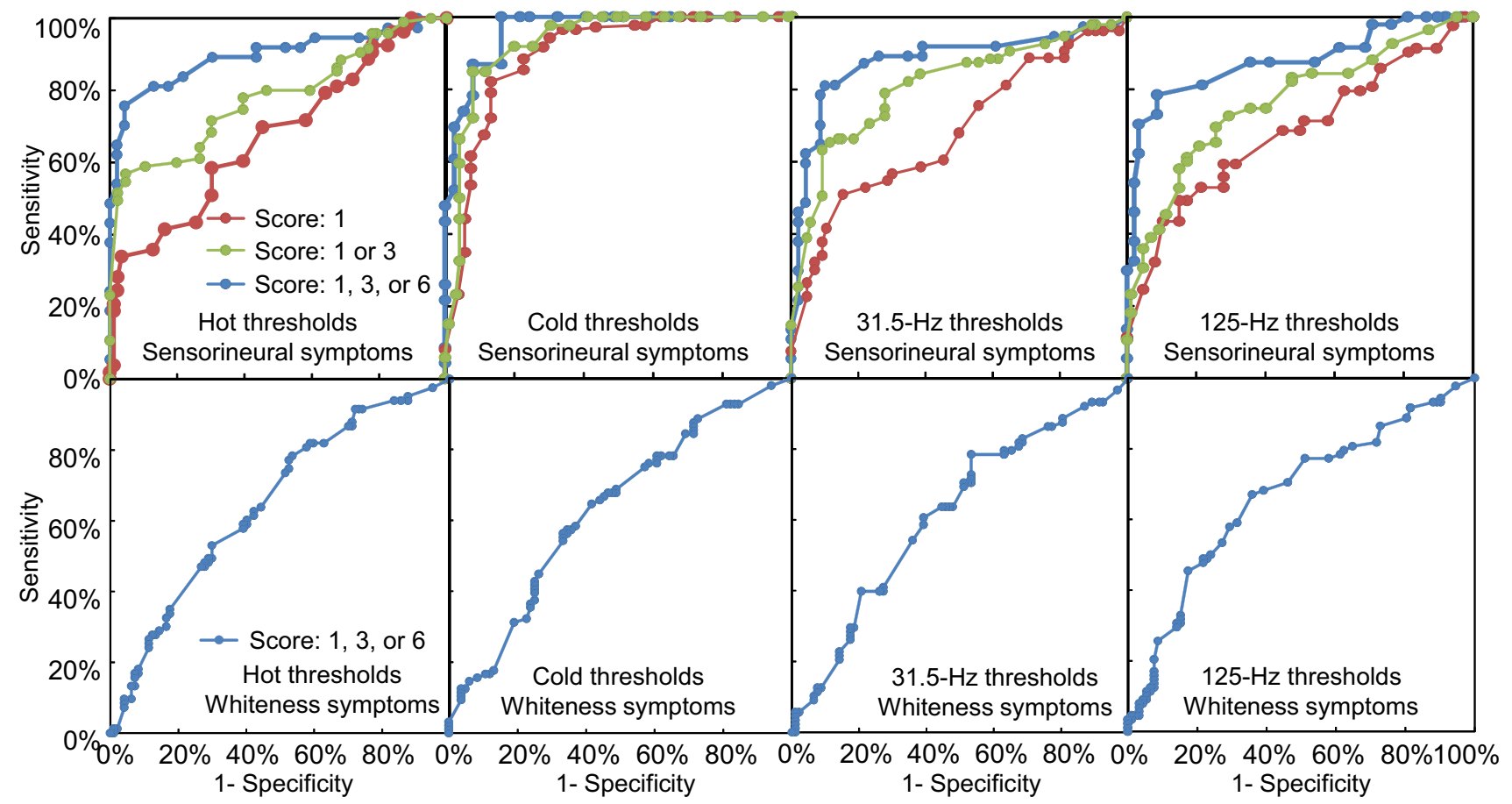

Fig. 1 Receiver operating characteristics (ROC) curves for sensorineural symptoms (top) and finger whiteness (bottom) calculated for hot and cold thresholds and vibrotactile thresholds at 31.5 and

for 31.5-Hz thresholds and 0.91 (CI of $0.85-0.97$ ) for $125-$ $\mathrm{Hz}$ thresholds (see Fig. 1). The diagnostic odds ratios and $95 \%$ confidence intervals for vibrotactile thresholds at 31.5 and $125 \mathrm{~Hz}$ were 39 (CI of 17-89) and 44 (CI of 19-101), respectively.

\section{Associations between thermotactile thresholds and vibrotactile thresholds}

For fingers with sensorineural scores of $0,1,3$, and 6, examples of associations between (a) hot and cold thresholds, (b) vibrotactile thresholds at 31.5 and $125 \mathrm{~Hz}$, (c) $31.5-\mathrm{Hz}$ thresholds and cold thresholds and (d) $31.5-\mathrm{Hz}$ thresholds and hot thresholds are shown in Fig. 2.

In Group A (with symptoms), there was a negative correlation between hot and cold thresholds on fingers with a sensorineural score of 6 ( $p=0.017-0.031$ ), but not on fingers with sensorineural scores of 1 or 3 ( $p=0.065-0.327$ ). In Group B (without symptoms), there was no statistically significant correlation between hot and cold thresholds on any of the four fingers ( $p=0.311-0.623)$.

In Group A, there was a positive correlation between vibrotactile thresholds at 31.5 and $125 \mathrm{~Hz}$ on fingers with a sensorineural score of $6(p<0.01)$, but not on fingers with sensorineural scores of 1 and 3 ( $p=0.094-0.169)$. In Group $\mathrm{B}$, there was a positive correlation between vibrotactile thresholds at 31.5 and $125 \mathrm{~Hz}$ on all four fingers $(p<0.05)$.
$125 \mathrm{~Hz}$. The ROC curves are shown for sensorineural scores of 1, 3, and 6 and for finger whiteness scores of 1,3, or 6 . Data from 60 subjects

In Group A, there were positive correlations between hot thresholds and vibrotactile thresholds (at both 31.5 and $125 \mathrm{~Hz}$ ) and negative correlations between cold thresholds and vibrotactile thresholds (at both 31.5 and $125 \mathrm{~Hz}$ ) on fingers with a sensorineural score of $6(p<0.05)$, except between cold thresholds and vibrotactile thresholds at $125 \mathrm{~Hz}(p=0.064-0.103)$. On fingers with a sensorineural score of 1 or 3 , there were no statistically significant correlations between thermotactile thresholds (hot or cold) and vibrotactile thresholds (at 31.5 or $125 \mathrm{~Hz})(p>0.1)$. In Group B, the thermotactile thresholds (for hot and cold) and vibrotactile thresholds (at 31.5 and $125 \mathrm{~Hz}$ ) were not significantly correlated with each other on any of the four fingers $(p=0.196-0.350)$.

\section{Associations between thresholds and HAVS symptoms (numbness, tingling, and whiteness)}

The sensitivity, the specificity, and the AUC were calculated for three reported HAVS symptoms (numbness, tingling, and whiteness) with four thresholds (hot and cold thresholds, vibrotactile thresholds at 31.5 and $125 \mathrm{~Hz}$ ). The findings summarised in Table 4 for the total of 240 fingers were obtained where a symptom corresponded to a score of 1 or greater.

Statistical analysis was also performed on thresholds between fingers with and without any blanching. There 

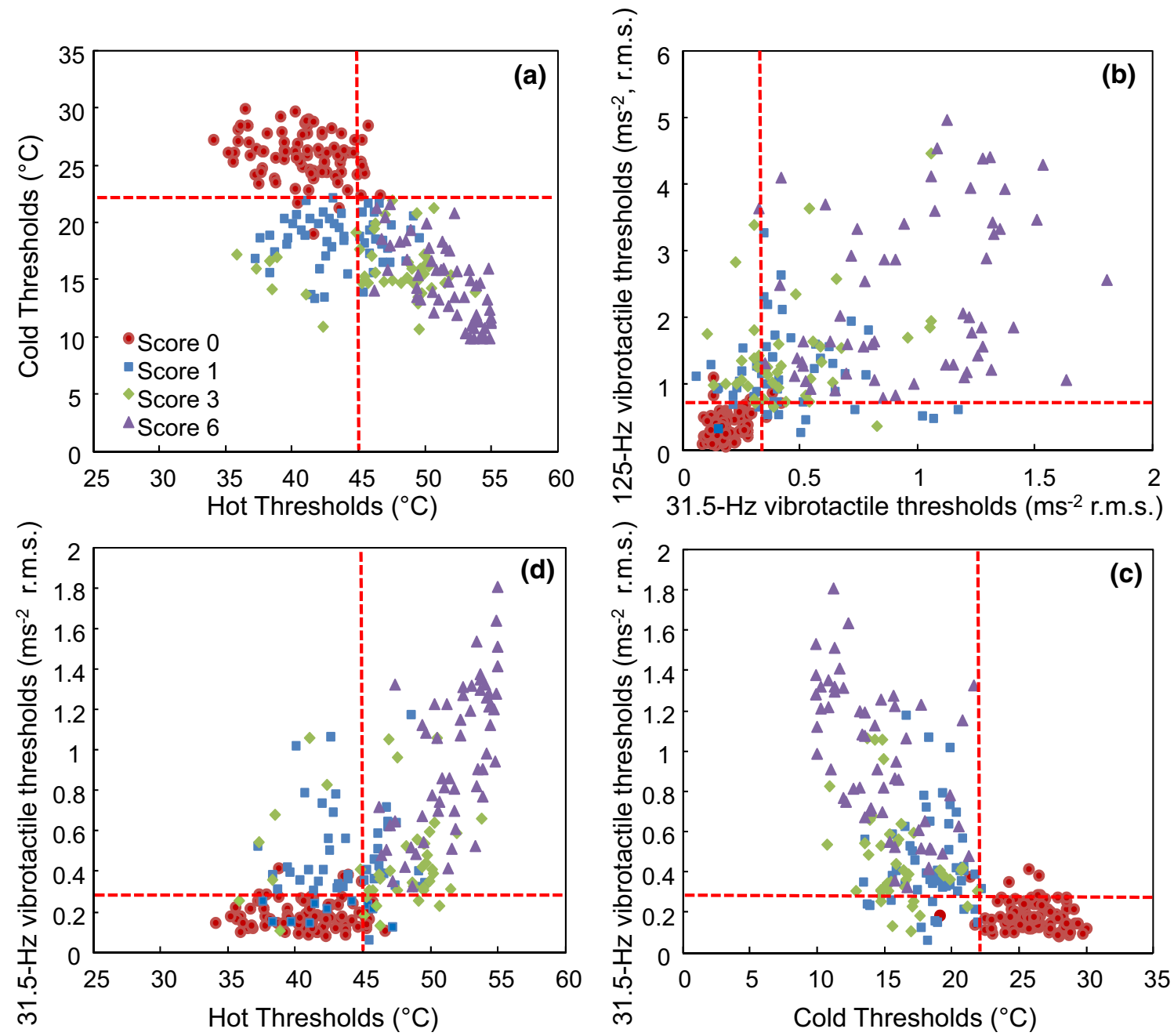

Fig. 2 Associations between a hot and cold thresholds, b vibrotactile thresholds at 31.5 and $125 \mathrm{~Hz}$, c vibrotactile thresholds at $31.5 \mathrm{~Hz}$ and cold thresholds and $\mathbf{d}$ vibrotactile thresholds at 31.5 and

hot thresholds in fingers with sensorineural scores of $0,1,3$, and 6 . Broken lines indicate diagnostic criteria for possible dysfunction (see Table 2). Data from 60 subjects

Table 4 Sensitivity, specificity, and area under the ROC curve (AUC) calculated for thermotactile and vibrotactile thresholds using symptoms of numbness, tingling, and whiteness

\begin{tabular}{|c|c|c|c|c|c|c|c|c|c|}
\hline \multirow[t]{2}{*}{ Measure } & \multicolumn{3}{|c|}{ Numbness } & \multicolumn{3}{|l|}{ Tingling } & \multicolumn{3}{|c|}{ Whiteness } \\
\hline & Sen. $(\%)$ & Spec. $(\%)$ & AUC & Sen. $(\%)$ & Spec. $(\%)$ & AUC & Sen. $(\%)$ & Spec. $(\%)$ & AUC \\
\hline Hot thresholds & 80 & 91 & 0.88 & 76 & 83 & 0.82 & 53 & 65 & 0.60 \\
\hline Cold thresholds & 85 & 92 & 0.94 & 82 & 88 & 0.87 & 50 & 63 & 0.59 \\
\hline Vibrotactile thresholds at $31.5 \mathrm{~Hz}$ & 77 & 88 & 0.85 & 73 & 85 & 0.82 & 49 & 64 & 0.58 \\
\hline Vibrotactile thresholds at $125 \mathrm{~Hz}$ & 79 & 89 & 0.86 & 72 & 83 & 0.82 & 52 & 68 & 0.64 \\
\hline
\end{tabular}

were significantly lower thresholds in fingers with blanching $(p<0.05)$, but no difference in any of the four thresholds between fingers with blanching scores of 1,3 , and 6 $(p=0.079-0.331)$.

\section{Discussion}

\section{Thermotactile thresholds}

In the present study, when using the tests and criteria currently employed in the UK, thermal perception thresholds provided useful indications of whether fingers had 
sensorineural symptoms: $81 \%$ sensitivity and $92 \%$ specificity for hot thresholds and $87 \%$ sensitivity and $94 \%$ specificity for cold thresholds. Thermal dysfunction was observed in digits innervated by both the median nerve (index finger) and the ulnar nerve (little finger). The findings are consistent with previous studies of thermal dysfunction in the fingers of various groups of vibration-exposed workers, including users of chain saws, hand-held grinders, and hammer drills (e.g. Virokannas and Virokannas 1995; Lindsell and Griffin 1999; Toibana et al. 2000; Nilsson and Lundström 2001; Sakakibara et al. 2002; Bovenzi et al. 2011).

Although conventional electro-neurophysiological methods cannot measure the anatomical integrity of small-calibre nerve fibres, their functional capacity can be assessed using a thermal aesthesiometer (Magda et al. 2002; Nilsson et al. 2008). However, there is no international standard for the measurement and evaluation of thermal thresholds and only a few studies have provided normative data for thermotactile thresholds in a healthy population (e.g. Nilsson et al. 2008; Seah and Griffin 2008). In the current study, hot and cold thresholds on fingers without sensorineural symptoms were similar to those reported previously for the same instrument and methodology (i.e. the same contact conditions, psychophysical method, reference temperature, and rate of change in temperature) (Lindsell and Griffin 1999; Seah and Griffin 2008; Bovenzi et al. 2011).

In the ROC curves, the greater the central portion of a curve moves upwards and to the left, the greater a measure distinguishes between fingers with and without sensorineural symptoms. In the ROC curve analysis, AUCs of 0.8 and 0.65 are described as having, respectively, 'good' and 'fair' discriminative ability (Weinstein and Fineberg 1980). For the hot and cold thresholds, the AUC was 0.89 and 0.96 , respectively, in the current study, indicating the thermotactile thresholds provided 'good' indications of whether a finger had sensorineural symptoms.

Some studies have concluded that the neutral zone between hot and cold thresholds was a sensitive indicator of nerve damage (Hirosawa et al. 1983; Ekenvall et al. 1986), while others have concluded that cold thresholds were more useful than hot thresholds in the detection of vibrationinduced neuropathy (Ekenvall et al. 1986; Virokannas and Virokannas 1995). Nilsson et al. (2008) reported reduced perception to cold but no significant changes in hot thresholds among young adults exposed to hand-transmitted vibration. In the present study, although greater sensitivity and greater specificity were obtained with cold thresholds, the difference was small and the patients appear to have been damaged similarly in their hot and cold receptors or neurological pathways. In patients with severe neurological injury, both the hot and the cold thresholds may be abnormal, whereas in patients with less injury one thermotactile channel may be affected than the other. Differences in the method of measuring thermal thresholds may also affect which channel appears to be most affected, as thermotactile thresholds depend on many factors including the starting temperature, the area of contact and the contact location (Hilz et al. 1995; Ruffell and Griffin 1995; Seah and Griffin 2010).

\section{Vibrotactile thresholds}

Vibrotactile thresholds at 31.5 and $125 \mathrm{~Hz}$ reflect the responses of two different mechanoreceptors in the skin and their afferent fibres (ISO 13091-1 2001). This study suggests vibrotactile thresholds can be powerful indicators of sensorineural symptoms of HAVS. They had sensitivities around $80 \%$ and specificities around $90 \%$ with an AUC greater than 0.9 . This is consistent with previous investigations, suggesting vibration perception thresholds can be used to assess sensory loss in the fingers of vibration-exposed workers with a history of sensorineural symptoms (e.g. Ekenvall et al. 1986, 1989; Virokannas and Virokannas 1995; Lundström et al. 1999; Bovenzi et al. 2011). Vibrotactile thresholds on fingers without sensorineural symptoms were consistent with those previously measured at other European test centres using the same measurement apparatus, psychophysical methods, and skin-stimulus contact conditions (Lindsell and Griffin 2003). Although clinical electrophysiology may be used to assess large fibre functions, vibrotactile thresholds have been reported to be more sensitive than conventional neurography (Rolke et al. 2006).

\section{Association between thermotactile thresholds and vibrotactile thresholds}

On fingers with more severe symptoms (i.e. sensorineural score of 6), thermotactile thresholds were correlated with vibrotactile thresholds. On fingers with less extensive sensorineural symptoms (sensorineural scores of 1 and 3), the four thresholds (i.e. hot and cold thresholds and vibrotactile thresholds at 31.5 and $125 \mathrm{~Hz}$ ) were not correlated with each other. This is partially consistent with Toibana et al. (2000) who found a significant correlation between thermal thresholds and pain thresholds but not between thermal thresholds and vibrotactile thresholds. The neural pathways for these four sensory systems differ: whereas the perception of vibration is mediated by the responses of Meissner's and Pacinian corpuscles and large-diameter myelinated A $\alpha$ afferent nerve fibres, the perception of warmth is mediated by thinly myelinated $\mathrm{A} \delta$ nerve fibres and the perception of coolness is mediated by unmyelinated $\mathrm{C}$ nerve fibres (Burgess and Perl 1973). Every nerve in the peripheral system has a specific function, so the signs and symptoms depend on the type of nerve affected. The functions of different peripheral receptors, neural pathways, and components of the sensory cortex are therefore evaluated by measuring the different 
thermotactile and vibrotactile thresholds. In some cases, a finger with a sensorineural score of 1 had reduced sensitivity to cold but had a normal hot threshold and normal vibrotactile thresholds. The absence of a statistically significant correlation between hot and cold thresholds on fingers with symptoms (numbness or tingling) reported on the distal and middle phalanges of a finger indicates the need for separate tests for hot and cold thresholds, rather than a single measure of the 'neutral zone'.

Cold thresholds had greater sensitivity and specificity than either hot thresholds or vibrotactile thresholds in fingers with mild symptoms (with sensorineural score of 1), consistent with reduced sensitivity to cold reported among young adults exposed to hand-transmitted vibration (Nilsson et al. 2008). Some studies of patients with the hand-arm vibration syndrome have concluded that thresholds for the perception of hot and cold temperatures are impaired before vibrotactile thresholds (Ekenvall et al. 1989; Virokannas and Virokannas 1995; Bovenzi et al. 2011), consistent with alterations in the thin non-myelinated fibres in nerve tissues of rats after exposure to vibration (Lundborg et al. 1990).

The cross-sectional design of the present study did not allow the investigation of changes in the signs and symptoms of sensory function in fingers over time- - this requires a longitudinal study of the relation between vibration exposure and sensory function. The study did not, therefore, seek to investigate the cause of the symptoms or signs and merely investigate differences in thresholds between fingers with and without sensorineural symptoms.

The study also found that thermotactile thresholds and vibrotactile thresholds were more impaired among patients with moderate and severe sensorineural symptoms, consistent with studies suggesting that thermotactile and vibrotactile thresholds can reflect the severity of nerve damage (Hirosawa et al. 1983; Toibana et al. 2000; Poole et al. 2016).

For all four thresholds, there was greater sensitivity and greater specificity for numbness than for tingling (Table 4), although patients with either numbness or tingling are likely to have impaired thermotactile and vibrotactile thresholds. Although the numbness and tingling symptoms were not identically distributed, the affected fingers were similar, so the study does establish which signs corroborate which symptoms.

Lower thresholds were found on fingers with blanching, but the sensitivity and specificity of thresholds for the detection of blanching were low (around 55\%). On fingers with blanching, there were no significant differences in thresholds between fingers with different blanching scores. So neither thermotactile thresholds nor vibrotactile thresholds were useful indicators of the presence or the severity of vascular dysfunction - the small association between thresholds and blanching may arise because both are caused by exposure to hand-transmitted vibration. The findings are consistent with
Ekenvall et al. (1989) who found no relation between vascular symptoms and the outcome of sensory testing. Whereas the chronic changes giving rise to finger blanching are not clearly associated with thresholds, acute reductions in finger blood flow caused by vibration are associated with thresholds for the perception of vibration (Ye and Griffin 2011, 2014, 2016b). The contradiction implies that the acute and chronic changes have different mechanisms, with the vascular impairment in vibration-exposed persons being associated with either a different form of neurological damage or some form of local structural damage causing the vascular phenomenon known as vibration-induced white finger.

The current study was conducted within patients referred for Tier 5 HAVS assessment. According to the tiered system used in UK, prior to the test, all patients will have been interviewed by an occupational physician who considered it likely the patient had HAVS, but recommended objective tests before confirming the decision. This means the patients were pre-selected by various occupational physicians, and all patients were likely to have at least one of the HAVS symptoms (numbness, tingling, or whiteness) on at least one finger. The sensitivities and specificities found in this study can be expected to be dependent on the population studied, including the extent to which patients had been exposed to hand-transmitted vibration, had relevant symptoms, or had other conditions that could affect either the symptoms or the signs of disorder. Although limitations to the testing time meant that only two fingers were tested on each hand, the finding of associations between the symptoms and signs on a finger should encourage the measurement of thresholds on all five fingers of both hands

Self-reported numbness and tingling scores can be overestimated or underestimated, but it is unlikely this was a major issue since the sensitivity and specificity show that thresholds were 'good' indicators of whether a finger had sensorineural symptoms. Furthermore, errors in reporting numbness and tingling would not have affected the relative performance of the four threshold tests. During interview with the patients, they were asked how often they experienced numbness and tingling (e.g. few times a year or few times a day), the duration of the symptoms (e.g. transient or permanent), and the conditions in which the symptoms occurred (e.g. during or after using vibrating tools, in cold condition). A single exposure to vibration may cause a temporary period of numbness or tingling that might be accompanied by impaired sensory perception, so it might be considered 'normal' for vibration-exposed workers to have intermittent numbness or tingling. Away from work, and when tested in a clinic, such temporary effects will have passed, so it seems reasonable to exclude them when judging the symptoms and when relating symptoms to signs. Temporary numbness or tingling was excluded in this study, but this does not imply that temporary effects are of no interest 
as they might be indicators of the likelihood of more permanent effects.

Impaired thermotactile or vibrotactile perception in a vibration-exposed worker is not a sufficient sign to diagnose the sensorineural component of the hand-arm vibration syndrome. Elevated thresholds in a finger should be considered in relation to a history of exposure to hand-transmitted vibration, symptoms in the same finger, and absence of alternative explanations of the signs and symptoms. Reduced sensitivity to temperature or vibration could imply damage to peripheral nerves caused by various conditions: traumatic injuries, infections, metabolic problems, diabetes mellitus or exposure to chemicals or toxins (Hughes 2002). With increased age, healthy men and women have been reported to have reduced sensitivity to both temperature (e.g. Bartlett et al. 1998; Lindsell and Griffin 2002) and vibration (Bartlett et al. 1998; Wild et al. 2001). The influences of general health, alcohol consumption, smoking, gender, profession, and age on thermotactile and vibrotactile thresholds merit greater attention.

\section{Conclusions}

For the conditions and protocols applied in the present study, both thermotactile thresholds and vibrotactile thresholds differed between fingers with and without sensorineural symptoms. Cold threshold had greater sensitivity and greater specificity on fingers with numbness or tingling scores of 1 , suggesting cold thresholds provide better indications of early sensorineural disorder.

\section{Compliance with ethical standards}

Conflict of interest The authors declare that they have no conflict of interest.

Open Access This article is distributed under the terms of the Creative Commons Attribution 4.0 International License (http://creativecommons.org/licenses/by/4.0/), which permits unrestricted use, distribution, and reproduction in any medium, provided you give appropriate credit to the original author(s) and the source, provide a link to the Creative Commons license, and indicate if changes were made.

\section{References}

Anonymous (1995) Clinical and laboratory diagnostics of neurological disturbances in workers using hand-held vibrating tools. Report from discussions in a working group. In: Gemne G, Brammer AJ, Hagberg M, Lundström R, Nilsson T (eds) Proceedings of the Stockholm Workshop 94. Hand-arm vibration syndrome: diagnostics and quantitative relationships to exposure. National Institute of Occupational Health, Solna, Sweden, 25-28 May 1994. Arb Hälsa vol 5, pp 187-194
Bartlett G, Stewart JD, Tamblyn R, Abrahamowicz M (1998) Normal distributions of thermal and vibration sensory thresholds. Muscle Nerve 21(3):367-374

Bovenzi M, Ronchese F, Mauro M (2011) A longitudinal study of peripheral sensory function in vibration-exposed workers. Int Arch Occup Environ Health 84:325-334

Brammer AJ, Taylor W, Lundborg G (1987) Sensorineural stages of the hand-arm vibration syndrome. Scand J Work Environ Health 13:279-283

Burgess PR, Perl ER (1973) Cutaneous mechanoreceptors and nociceptors. In: Iggo A (ed) Handbook of sensory physiology. Springer, Berlin, pp 29-78

Chong PST, Cros DP (2004) Technology literature review: quantitative sensory testing. Muscle Nerve 29:734-747

Dyck PJ, Karnes J, O’Brien PC, Zimmerman IR (1993) Detection thresholds of cutaneous sensation in humans. In: Dyck PJ (ed) Peripheral neurophysiology, vol 1. Publ. W. B. Saunders, Philadelphia. ISBN: 0-7216-3273-4

Ekenvall L, Nilsson BY, Gustavsson P (1986) Temperature and vibration threshold in vibration syndrome. Br J Ind Med 43:825-829

Ekenvall L, Gemne G, Tegner R (1989) Correspondence between neurological symptoms and outcome of quantitative sensory testing in the hand-arm vibration syndrome. $\mathrm{Br} \mathrm{J}$ Ind Med 46:570-574

Gemne G, Pyykko I, Taylor W, Pelmear PL (1987) The Stockholm Workshop Scale for the classification of cold-induced Raynaud's phenomenon in the hand arm vibration syndrome. Scand J Environ Health 13:275-278

Griffin MJ (1990) Handbook of human vibration. Academic, London

Griffin MJ (2008) Measurement, evaluation, and assessment of peripheral neurological disorders caused by hand-transmitted vibration. Int Arch Occup Environ Health 81:559-573

Health and Safety Executive (2005) Hand-arm vibration: the control of vibration at work regulations 2005 and guidance on regulations. HSE Books, London

Hilz MJ, Glorius S, Beric A (1995) Thermal perception thresholds: influence of determination paradigm and reference temperature. J Neurol Sci 129:135-140

Hirosawa I, Nishiyama K, Watanabe S (1992) Temporary threshold shift of temperature sensation caused by vibration exposure. Int Arch Occup Environ Health 63:531-535

Hirosawa I, Watanabe S, Fukuchi Y, Nishiyama K, Hosokawa M (1983) Availability of temperature sense indices for diagnosis of vibration disease. Int Arch Occup Environ Health 52(3):215-222

Hughes RAC (2002) Clinical review: peripheral neuropathy. Br Med J 324:466-469

International Organization for Standardization (ISO) (2001) Mechanical vibration-vibrotactile perception thresholds for the assessment of nerve dysfunction-Part 1: Methods of measurement at the fingertips. ISO 13091-1. ISO, Geneva

International Organization for Standardization (ISO) (2003) Mechanical vibration-vibrotactile perception thresholds for the assessment of nerve dysfunction-Part 2: Analysis and interpretation of measurements at the fingertips. ISO 13091-2. ISO, Geneva

Lindsell C, Griffin MJ (1998) Standardised diagnostic methods for assessing components of the hand-arm vibration syndrome. CRR 197/1998. HSE Books, Sudbury, Suffolk, pp 1-87. ISBN 0-7176-1640-1

Lindsell CJ, Griffin MJ (1999) Thermal thresholds, vibrotactile thresholds, and finger systolic blood pressures in dockyard workers exposed to hand-transmitted vibration. Int Arch Occup Environ Health 72:377-386

Lindsell CJ, Griffin MJ (2002) Normative data for vascular and neurological test of the hand-arm vibration syndrome. Int Arch Occup Environ Health 75:43-54 
Lindsell CJ, Griffin MJ (2003) Normative vibrotactile thresholds measured at five European test centres. Int Arch Occup Environ Health 76:517-528

Lundborg G, Dahlin LB, Hansson H-A, Kanje M, Necking L-E (1990) Vibration exposure and peripheral nerve fiber damage. J Hand Surg 15A:346-351

Lundström R, Nilsson T, Burström L, Hagberg M (1999) Exposureresponse relationship between hand-arm vibration and vibrotactile perception sensitivity. Am J Ind Med 35:456-464

Magda P, Latov N, Renard MV, Sander HW (2002) Quantitative sensory testing: high sensitivity in small fiber neuropathy with normal NCS/EMG. J Peripher Nerv Syst 7:225-228

Nilsson T, Lundström R (2001) Quantitative thermal perception thresholds relative to exposure to vibration. Occup Environ Med 58:472-478

Nilsson T, Burström L, Hagberg M, Lundström R (2008) Thermal perception thresholds among young adults exposed to hand transmitted vibration. Int Arch Occup Environ Health 81:519-533

Poole CJM, Mason H, Harding AH (2016) The relationship between clinical and standardized tests for hand-arm vibration syndrome. Occup Med 66:285-291

Rolke R, Magerl W, Campbell KA, Schalber C, Caspari S, Birklein F, Treede R-D (2006) Quantitative sensory testing: a comprehensive protocol for clinical trials. Eur J Pain 10:77-88

Ruffell CM, Griffin MJ (1995) Effect of starting temperature on the repeatability of thermotactile thresholds. Cent Eur J Public Health 3(Suppl):81-84

Sakakibara H, Maeda S, Yonekawa Y (2002) Thermotactile threshold testing for the evaluation of sensory nerve function in vibration exposed patients and workers. Int Arch Occup Environ Health 75:90-96

Seah SA, Griffin MJ (2008) Normal values for thermotactile and vibrotactile thresholds in males and females. Int Arch Occup Environ Health 81:535-543

Seah SA, Griffin MJ (2010) Thermotactile thresholds at the fingertip: effect of contact area and contact location. Somatosens Mot Res 27(3):82-92
Shy ME, Frohman EM, So YT, Arezzo JC, Cornblath DR, Giuliani MJ, Kincaid JC, Ochoa JL, Parry GJ, Weimer LH (2003) Quantitative sensory testing. Report of the therapeutics and technology assessment subcommittee of the American Academy of Neurology. Neurology 60:898-904

Toibana N, Sakakibara H, Hirata M, Kondo T, Toyoshima H (2000) Thermal perception threshold testing for the evaluation of small sensory nerve fiber injury in patients with hand-arm vibration syndrome. Ind Health 38:366-371

Virokannas H, Virokannas A (1995) Temperature and vibration perception thresholds in workers exposed to hand-arm vibration. Cent Eur J Public Health 3(Suppl):66-69

Weinstein MC, Fineberg HV (1980) Clinical decision analysis. W. B. Saunders, Philadelphia

Wild P, Massin N, Lasfargues G, Baudin V, Unlu D, Donati P (2001) Vibrotactile perception thresholds in four non-exposed populations of working age. J Ergon 44(6):649-657

Ye Y, Griffin MJ (2011) Reductions in finger blood flow in men and women induced by $125-\mathrm{Hz}$ vibration: association with vibration perception thresholds. J Appl Physiol 111:1606-1613

Ye Y, Griffin MJ (2014) Relation between vibrotactile perception thresholds and reductions in finger blood flow induced by vibration of the hand at frequencies in the range $8-250 \mathrm{~Hz}$. Eur J Appl Physiol 114(8):1591-1603

Ye Y, Griffin MJ (2016a) Assessment of two alternative standardised tests for the vascular component of the hand-arm vibration syndrome (HAVS). Occup Environ Med 73:701-708. doi:10.1136/ oemed-2016-103688

Ye Y, Griffin MJ (2016b) Reductions in finger blood flow induced by $125-\mathrm{Hz}$ vibration: effect of location of contact with vibration. Int Arch Occup Environ Health 89:425-433

Zaslansky R, Yarnitsky D (1998) Clinical applications of quantitative sensory testing (QST). J Neurol Sci 153:215-238 